\title{
Angiogenesis of Oral Lichen Planus: A possible pathogenetic mechanism
}

\author{
Giuseppe-Alessandro Scardina, Alessia Ruggieri, Pietro Messina, Emiliano Maresi
}

University of Palermo Department of Oral Sciences “G. Messina”. Palermo (Italy)

\author{
Correspondence: \\ University of Palermo \\ Department of Oral Sciences "G. Messina” \\ Via Del Vespro, 129 \\ 90127 Palermo (ITALY). \\ scardina@odonto.unipa.it
}

Received: $10 / 12 / 2008$

Accepted: $20 / 05 / 2009$

\author{
Scardina GA, Ruggieri A, Messina P, Maresi E. Angiogenesis of Oral \\ Lichen Planus: A possible pathogenetic mechanism. Med Oral Patol Oral \\ Cir Bucal. 2009 Nov 1;14 (11):e558-62. \\ http://www.medicinaoral.com/medoralfree01/v14i11/medoralv14i11p558.pdf \\ Article Number: $2609 \quad$ http://www.medicinaoral.com \\ (C) Medicina Oral S. L. C.I.F. B 96689336 - pISSN $1698-4447$ - eISSN: 1698-6946 \\ eMail: medicina@medicinaoral.com \\ Indexed in: \\ -SCI EXPANDED \\ -JOURNAL CITATION REPORTS \\ -Index Medicus / MEDLINE / PubMed \\ -EMBASE, Excerpta Medica \\ -SCOPUS \\ -Indice Médico Español
}

\begin{abstract}
Objective: Oral Lichen Planus (OLP) is a chronic inflammatory disease with an autoimmune inflammatory pathogenesis. The aim of the research is to compare the vascular endothelial growth factor (VEGF) and adhesion of molecules in the biopsy samples of patients affected by OLP, in order to research the presence of the angiogenetic phenomenon and to understand its pathogenetic mechanism. Materials and Methods: Thirty OLP patients and thirty healthy subjects were enrolled in a study. The immunohistochemical analysis of the VEGF and vascularendothelial adhesion molecules was carried out by means of primary antibodies and anti-CD34, anti-VEGF, antiCD106 antigen (VCAM-1) and anti-CD54 antigen (ICAM-1). The statistical significance of the differences was checked with the Mann-Whitney test (MW test). The level of significance was set to $\mathrm{P}<0.001$. Data analysis was carried out with StatView 5.0.1 (SAS Institute Inc., Cary, NC). Results: The results reveal the presence of a significant angiogenesis in OLP patients for the VEGF, CD34, CD106 and CD54 $(\mathrm{P}<0.001)$.. The number of vessels in the biopsies of the patients with OLP (mean \pm SD: $21.27 \pm 4.85$ ), compared with the healthy subjects (mean \pm SD: $4.74 \pm 0.97$ ) was significantly more (Mann-Whitney test, $\mathrm{P}<0.001$ ). The positive expression rate of VEGF, CD34, VCAM-1 and ICAM-1 in oral lichen samples was $64.2 \%, 54.3 \%, 32.5 \%$ and $29.7 \%$, respectively. Isolated endothelial cells and newly-formed micro-vessels and endothelial cells with high-immune-positivity to the antibodies anti-ICAM-1 and anti-VCAM-1 were observed. Conclusions: The results of our immunohistochemical research show that a significant neoangiogenesis occurs in oral lichen planus.
\end{abstract}

Key words: Oral Lichen Planus, angiogenesis, immunohistochemical, VEGF, VCAM-1, ICAM-1.

\section{Introduction}

Angiogenesis represents a cycle of vital processes that leads to the neoformation of anomalous blood vessels in pre-existing vascular structures (1). At the heart of angiogenesis is the endothelial cell that proliferates and differentiates under the regulatory action of vascular endothelial growth factor (VEGF), the principal direct inducer of angiogenesis together with other growth cofac- tors. This process plays an important role both in physiological conditions, such as embryonic development and the healing of wounds, and in pathological conditions, such as the growth of tumours and metastasis and the development of chronic inflammatory diseases. Scientific studies (2-4) have shown the importance of angiogenesis in the chronic inflammatory mechanism and have verified the presence of neoangiogenesis in pathologies 
such as rheumatoid arthritis, psoriasis, bronchial asthma, diabetic retinopathy, atherosclerosis and Alzheimer's disease. Furthermore, recent studies (5) have confirmed a strong involvement of the angiogenetic phenomenon in other intestinal chronic inflammatory diseases such as Crohn's Disease and ulcerous rectocolitis. It is known that angiogenesis plays a key role in the pathogenesis of chronic inflammatory illnesses, not only causing the gemmation of new vessels that allow a better oxygenation and a greater contribution of metabolites to the proliferating tissue, but also notably increasing the complex system of feed-back and turnover of the cells involved in the inflammatory process (6). This feed-back mechanism has been noticed (5) the presence - in isolated endothelial cells - of the prominent factor in the angiogenetic phenomenon: VEGF. Oral lichen planus (OLP) is a pathology of the oral mucous of a chronic inflammatory autoimmune pathogenetic mechanism in which a rich vascular proliferation has been seen, probably as a reaction to the hypoxic effect on the stromal area, where the phlogistic reaction occurs due to the increase of proliferating lymphocytes (7-8). The markers VEGF, CD34,VCAM-1 and ICAM-1 are appropriate to investigate the vascular endothelin and they have been used in the study to probe the presence of angiogenesis process and to underline endothelial modifications (9-10). In this research we evaluated the presence of the angiogenetic phenomenon in a series of biopsies of the oral mucous of patients with an anatomo-clinical diagnosis of lichen planus, with the aim of understanding its pathogenetic mechanism.

\section{Materials and Methods}

ThirtyOLPpatients(M/F:24/6; mean \pm SD:54.8 \pm 0.23 ) and thirty healthy subjects (M/F: 22/8; mean \pm SD:52.5 \pm 0.57 ) were enrolled. The diagnosis of lichen planus has been effected according to the WHO diagnostic criteria of oral lichen planus. These criteria are based on clinical and histopathological characteristics. Clinical criteria are: presence of bilateral, more or less symmetrical lesions; presence of a lacelike network of slightly raised graywhite lines (reticular pattern); erosive, atrophic, bullous and plaque-type lesions are only accepted as a subtype in the presence of reticular lesions else where in the oral mucosa. Histopathological criteria are: presence of a well-defined band-like zone of cellular infiltration that is confined to the superficial part of the connective tissue, consisting mainly of lymphocytes; Signs of 'liquefaction degeneration' in the basal cell layer; absence of epithelial dysplasia (11). Every patient which had lichenoid lesions objectively related to esogenic agents such as dental materials, medicines or specific allergens were excluded from the sample group (12). After having signed the informed consent the patients and the healthy subjects were submitted to an incisional biopsy through means of a biopsy punch ( $\varnothing=4 \mathrm{mms}$ ). All the bioptic samples were analyzed using the same procedures and materials. For each sample, lined up in paraffin after being fixed in formalin, four sections of tissue around $5 \mu \mathrm{ms}$ thick were prepared in order to enable an immunohistochemical analysis of the vascular endothelial growth factor (VEGF) and vascular-endothelial adhesion molecules in the vascular endothelial cells. These sections were treated with primary antibodies (DAKO and Novocastra) anti-CD34, anti-VEGF, anti-CD106 antigen (VCAM-1) and anti-CD54 antigen (ICAM-1). The dilution of the primary antibodies was: 1:500 for anti-VEGF and 1:50 for anti-CD106, anti-CD34 and anti CD-54. We have positive and negative immuunohistochemical controls for the different antibodies used.

The primary antibodies were revealed with the standard avidin-biotin method. Angiogenesis was valuated with a semiquantitative method counting for all the markers and in every histological section and in 5 random fields with high magnification (40X). The statistical significance of the differences was checked with the Mann-Whitney test (MW test). The level of significance was set to $\mathrm{P}<0.001$. Data analysis was carried out with StatView 5.0.1 (SAS Institute Inc., Cary, NC).

\section{Results}

The number of vessels in the biopsies of the patients with OLP (mean \pm SD: $21.27 \pm 4.85$ ), compared with the healthy subjects (mean \pm SD: $4.74 \pm 0.97$ ) was significantly increased (Mann-Whitney test, $\mathrm{P}<0.001$ ). The positive expression rate of VEGF, CD34, VCAM-1 and ICAM-1 in oral lichen samples were $64.2 \%, 54,3 \%, 32,5 \%$ and $29,7 \%$, respectively (Table 1). Moreover in the context of the phlogistic process and in a random way, both isolated endothelial cells immunopositive to the antibody anti-VEGF and neoformed microvessels and endothelial cells with high-immune-positivity to the antibodies antiICAM-1 and anti-VCAM-1 were observed (Figs 1,2). In healthy subjects we had positive immunohistochemical results for the CD34. Instead, we had negative immunohistochemical results for the other antibodies.

\section{Discussion}

The angiogenetic phenomenon is considered fundamental in many physiological and pathological processes (13). In any type of angiogenetic process, both physiological and pathological, the beginning of the angiogenic response occurs with the activation of the endothelial cells due to the release of cytokine following hypoxia or ischemia. For this reason, such conditions become a prerequisite for the participation of the endothelial cells in the process of angiogenesis. Hypoxic tumour cells, macrophages and other cells of the immune system produce VEGF that provokes vasodilation, the increase of vascular permeability and the release of proteasis, which 
Table 1. Conclusive results of the statistical analysis performed by Mann-Whitney U-test $(\mathrm{P}<.001)$.

\begin{tabular}{|l|c|c|}
\hline \multicolumn{1}{|c|}{ Age } & LPO patients & Healthy subjects \\
\hline (Mean \pm SD) & $54.8 \pm 0.23$ & $52.5 \pm 0.57$ \\
\hline Gender & $24 / F$ & $22 / F$ \\
\hline $\begin{array}{l}\text { Number of vessels } \\
\text { (Mean } \pm \text { SD) }\end{array}$ & $6 / M$ & $8 / M$ \\
\hline $\begin{array}{l}\text { Positive } \\
\text { Expression Rate } \\
\text { of Markers (\%) }\end{array}$ & VEGF: $64,2 \%$ & $4.74 \pm 0.97$ \\
\hline $\begin{array}{l}\text { Statistical Signifi- } \\
\text { cance }\end{array}$ & CD106 ( VCAM-1):32,5\% $54,3 \%$ & CD34: 18,3\% \\
(Mann Whitney test) & CD54 ( ICAM-1): $29,7 \%$ & \\
\hline
\end{tabular}
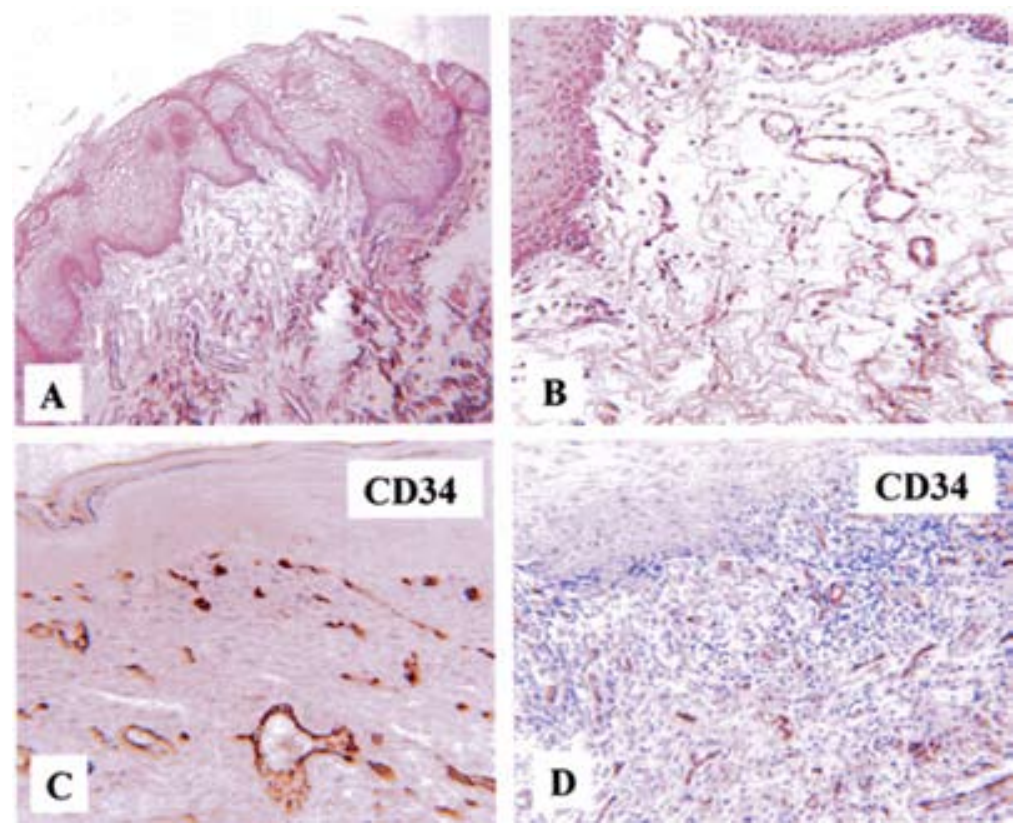

B

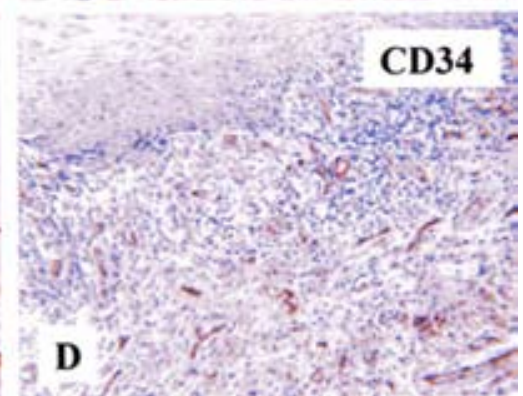

Fig. 1. A-B) Control: poor microcirculation subepithelially. C-D) Oral Lichen Planus: marked new-angiogenesis in lymphocytic infiltration (magnification 40X). 

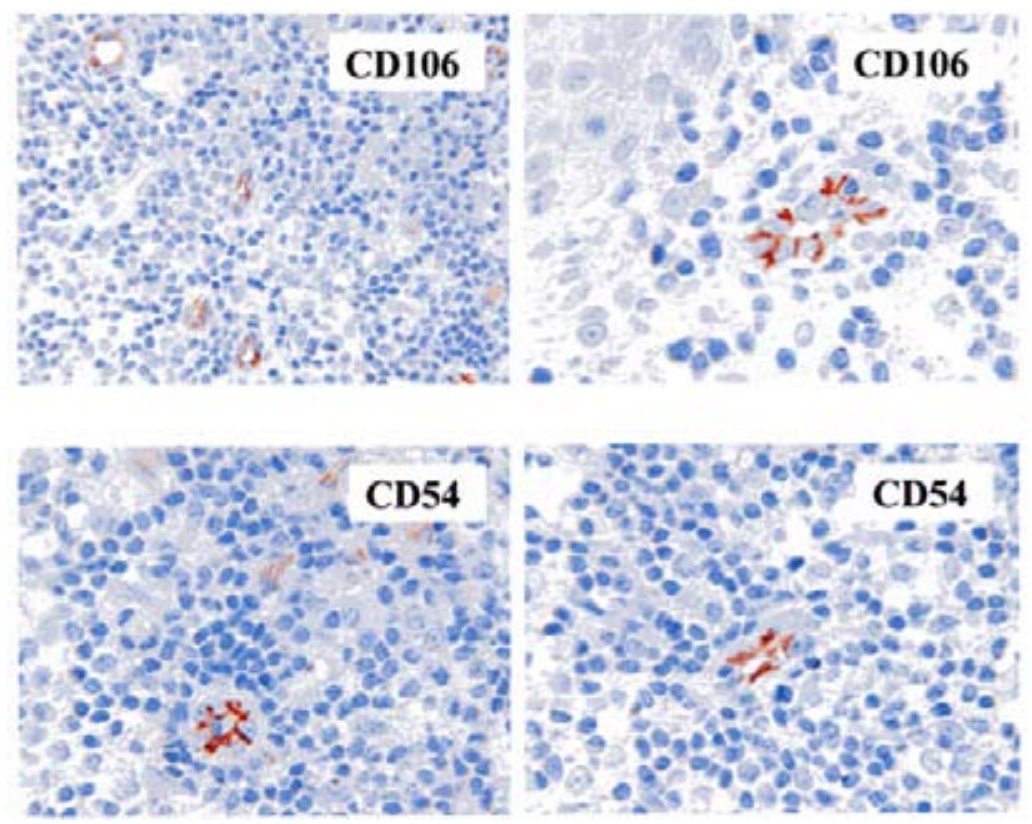

Fig. 2. Oral Lichen Planus: positivity of vessels neoformed to CD106 and CD54 antibodies cells (magnification 40X).

is important for the invasion of cells and the remodelling of tissues (14-16). VEGF has a fundamental role in the physiological and pathological regulation of angiogenesis. In vitro, VEGF stimulates the degradation, the proliferation and the migration of the endothelial cells. In vivo, it regulates vascular permeability, which is important for the start of angiogenesis. Different mechanisms are involved in the regulation of the gene for VEGF. The tissue tension of $\mathrm{O}_{2}$ plays an important role, as shown by the reversible increase of the levels of m-RNA for VEGF under conditions of hypoxia in vivo and in vitro (17). The literature is not, at the moment, satisfactory and up-to-date compared with the study of the angiogenetic phenomenon in OLP. As an autoimmune disease with an inflammatory origin and chronic progression, OLP satisfies all the prerequisites of hypoxia at the base of the angiogenetic mechanism in light of the hypoxic effect caused by the proliferating inflammatory elements that has also been found in the oral mucous with nonhistological or serological techniques, such as videocapillaroscopy (18). Studies effected on patients affected by OLP have had as their point of interest the increase of the number of mastocytes up to the level of the lamina itself. Such studies have proved how such an increase of density can, in effect, influence the vascular endothelia in a critical way and have an important role in the formation phase of the lesion (19). Immunohistochemical studies performed in the past have suggested that there is the intervention of the angiogenetic phenomenon in the malignant transformation of a lot of precancerous lesions of the oral epithelium, including OLP, but such studies were hypothetical and aimed at the oncologic consequence of the lesion (20).

Our research represents a direct assessment of the presence of the angiogenetic phenomenon in OLP since it is based on direct research and on histological samples of oral mucous, of VEGF - the principal factor for the angiogenetic process - and of other important factors of adhesion.

The assessment of the expression of the inducer factors of angiogenesis represents an important departure point for the study of new therapies based on the use of antiangiogenetic medicines already used in other pathologies with chronic inflammatory pathogenesis with good results. Moreover, the verified presence of angiogenesis in OLP could give further clarifications with respect to the ethiopathogenesis of OLP, which is still unknown today. In conclusion, the individualization of both lymphocytes in transit among the cubic endothelial cells of the neoformed vessels and adhesion molecules (antiVCAM-1 and anti-ICAM-1antibodies) in the endothelial cells of such vessels, suggests that angiogenesis in the lichen not only restores tissue oxygenation but also develops an important role in the turnover of the inflammatory elements in the inflamed area and therefore in the self-perpetuation of the disease. 


\section{References}

1. Liekens S, De Clercq E, Neyts J. Angiogenesis: regulators and clinical applications. Biochem Pharmacol. 2001;61:253-70.

2. Halin C, Detmar M. Chapter 1. Inflammation, angiogenesis, and lymphangiogenesis. Methods Enzymol. 2008:445:1-25.

3. Jakobsson L, Claesson-Welsh L. Vascular basement membrane components in angiogenesis--an act of balance. ScientificWorldJournal. 2008;8:1246-9.

4. Wirostko B, Wong TY, Simó R. Vascular endothelial growth factor and diabetic complications. Prog Retin Eye Res. 2008;27:608-21. 5. Pousa ID, Maté J, Gisbert JP. Angiogenesis in inflammatory bowel disease. Eur J Clin Invest. 2008;38:73-81.

6. Monaco C, Andreakos E, Kiriakidis S, Feldmann M, Paleolog E. T-cell-mediated signalling in immune, inflammatory and angiogenic processes: the cascade of events leading to inflammatory diseases. Curr Drug Targets Inflamm Allergy. 2004;3:35-42.

7. Sousa FA, Rosa LE. Oral lichen planus: clinical and histopathological considerations. Braz J Otorhinolaryngol. 2008;74:284-92.

8. Setterfield JF, Black MM, Challacombe SJ. The management of oral lichen planus. Clin Exp Dermatol. 2000;25:176-82.

9. Chimenos-Küstner E, Font-Costa I, López-López J. Oral cancer risk and molecular markers. Med Oral Patol Oral Cir Bucal. 2004;9:381-4, 377-80.

10. Griffioen AW. Anti-angiogenesis: making the tumor vulnerable to the immune system. Cancer Immunol Immunother. 2008;57:1553-8. 11. Van der Meij EH, Mast H, Van der Waal I. The possible premalignant character of oral lichen planus and oral lichenoid lesions: a prospective five-year follow-up study of 192 patients. Oral Oncol. 2007:43:742-8.

12. Thornhill MH, Sankar V, Xu XJ, Barrett AW, High AS, Odell EW, et al. The role of histopathological characteristics in distinguishing amalgam-associated oral lichenoid reactions and oral lichen planus. J Oral Pathol Med. 2006;35:233-40.

13. Van Beijnum JR, Fan TP, Griffioen AW. Eur(omega)conference Angiogenesis II. Angiogenesis. 2003;6:159-64.

14. Roskoski R Jr. VEGF receptor protein-tyrosine kinases: structure and regulation. Biochem Biophys Res Commun. 2008;375:287-91.

15. Prime SS, Davies M, Pring M, Paterson IC. The role of TGF-beta in epithelial malignancy and its relevance to the pathogenesis of oral cancer (part II). Crit Rev Oral Biol Med. 2004;15:337-47.

16. Prime SS, Pring M, Davies M, Paterson IC. TGF-beta signal transduction in oro-facial health and non-malignant disease (part I). Crit Rev Oral Biol Med. 2004;15:324-36.

17. Tipoe GL, Jin Y, White FH. The relationship between vascularity and cell proliferation in human normal and pathological lesions of the oral cheek epithelium. Eur J Cancer B Oral Oncol. 1996;32B:24-31. 18. Scardina GA, Picone V, Cacioppo A, Messina P. Study of microcirculation in oral lichen planus by video-capillaroscopy. Oral Surg Oral Med Oral Pathol Oral Radiol Endod. 2007;103:e30-4.

19. Zhao ZZ, Savage NW, Walsh LJ. Associations between mast cells and laminin in oral lichen planus. J Oral Pathol Med. 1998;27:163-7. 20. Jin Y, Tipoe GL, White FH, Yang L. A quantitative investigation of immunocytochemically stained blood vessels in normal, benign, premalignant and malignant human oral cheek epithelium. Virchows Arch. 1995;427:145-51. 